\title{
The Type III Effector AvrBsT Enhances Xanthomonas perforans Fitness in Field-Grown Tomato
}

\author{
Peter Abrahamian, Sujan Timilsina, Gerald V. Minsavage, Sushmita KC, Erica M. Goss, \\ Jeffrey B. Jones, and Gary E. Vallad ${ }^{\dagger}$
}

First, second, fourth, and seventh authors; Gulf Coast and Research Education Center, Wimauma, FL; first, second, third, fifth, sixth, and seventh authors: Department of Plant Pathology, University of Florida, Gainesville; and fifth author: Emerging Pathogens Institute, University of Florida, Gainesville.

Accepted for publication 11 June 2018.

\begin{abstract}
Type III secretion system effectors contribute to pathogenicity through various mechanisms. Recent surveys showed an increasing prevalence of the type III secretion effector avrBsT among Xanthomonas perforans strains. We hypothesized that the acquisition of $\operatorname{avrBs} T$ has a fitness advantage for the pathogen. The contribution of $a v r B s T$ to fitness on tomato was evaluated based on disease severity, in planta growth, competition, and recovery rates of wild-type (WT) and avrBsT mutant strains in greenhouse and field plants. GEV872 and GEV1001, representative strains of two phylogenomic groups of $X$. perforans, were selected for generating avrBsT mutants. Disease severity was higher for WT strains compared with the avrBsT mutant

strains. X. perforans WT and avrBsT mutant strains did not differ following leaf infiltration of greenhouse plants in direct competition and in planta growth assays. The effect of avrBsT on pathogen fitness was noticeable under field conditions. Differences in strain recovery were significant, with WT being recovered two to eight times more than avrBsT mutant strains in the case of both strains GEV872 and GEV1001. WT strains were capable of spreading longer distances across field plots compared with avrBsT mutant strains. Findings suggest that the functional AvrBsT affects the fitness of $X$. perforans under field conditions, making it an ideal candidate for bacterial spot resistance breeding efforts in tomato.
\end{abstract}

Plant-pathogenic bacteria have evolved diverse strategies to suppress plant defense responses (Galán and Collmer 1999). Most gram-negative plant-pathogenic bacteria rely on the type III secretion system (T3SS) to suppress plant defenses (Gürlebeck et al. 2006). The T3SS is encoded by a large conserved gene cluster termed hrp (Alfano and Collmer 2004). The hrp gene cluster is essential for maintaining pathogenicity in several gram-negative plant-pathogenic bacteria (Alfano and Collmer 2004). The T3SS secretes effector proteins into plant cells that are responsible for modulating cellular processes, mimicking host proteins, and contributing to virulence (Jacques et al. 2016)). Plant-pathogenic bacteria can acquire novel effectors mainly through horizontal gene transfer (Alfano and Collmer 2004; Barak et al. 2016).

Bacterial spot of tomato (BST) in Florida is caused primarily by Xanthomonas perforans (Potnis et al. 2015). BST leads to high yield losses in the field due to defoliation (Pohronezny et al. 1986). $X$. euvesicatoria was the only causal organism of BST in Florida through the early 1990s but was gradually replaced by the introduction of $X$. perforans race T3 strains (Jones et al. 1998). $X$. perforans race T4 is the prevalent cause of BST, and other BSTcausing Xanthomonas spp. such as X. euvesicatoria, X. gardneri, and $X$. vesicatoria were not recovered from tomato fields in Florida (Horvath et al. 2012; Schwartz et al. 2015; Stall et al. 2009). Phylogenomic analysis of a number of strains from recent surveys revealed the presence of two groups ( 1 and 2$)$ within the T4 race

†Corresponding author: G. E. Vallad: E-mail: gvallad@ufl.edu

Funding: This research is based upon work that is supported by the United States Department of Agriculture National Institute of Food and Agriculture under award number 2015-51181-24312.

*The $\boldsymbol{e}$-Xtra logo stands for "electronic extra" and indicates that two supplementary figures and four supplementary tables are published online.

(c) 2018 The American Phytopathological Society structure (Schwartz et al. 2015; Timilsina et al. 2015). Based on a comparative genomic study, the majority of the strains of both groups acquired a new effector, avrBsT (Schwartz et al. 2015; Timilsina et al. 2016). avrBsT was initially characterized from $X$. euvesicatoria in Early Cal Wonder (ECW) pepper plants (Minsavage et al. 1990). These X. euvesicatoria strains were pathogenic on tomato only and elicited a hypersensitive response (HR) on ECW pepper plants carrying the Bst resistance locus (Minsavage et al. 1990). avrBsT was initially identified in the first race T4 strain isolated in 1998 from Florida (Timilsina et al. 2016). Race T3 strains collected prior to 1998 did not carry avrBsT but T3 strains isolated later did (Timilsina et al. 2016). The presence of avrBsT in $X$. perforans limits its host range to tomato only (Minsavage et al. 1990). Furthermore, avrBsT is present in $X$. vesicatoria strains but not in X. gardneri (Kim et al. 2010; Schwartz et al. 2015).

AvrBsT (syn. XopJ2) is a plasmid-borne type III effector belonging to the XopJ clade within the YopJ superfamily of effectors that are present in human and plant bacterial pathogens (Ma and Ma 2016). The hallmark feature of this effector group is the presence of a conserved cysteine protease catalytic triad domain which is essential for cellular activity (Lewis et al. 2011; Orth et al. 2000). The XopJ effector group contains several characterized effectors such as XopJ1, AvrBsT (XopJ2), AvrRxv (XopJ3), AvrXv4 (XopJ4), and AvrXccB (XopJ5) (Büttner and Bonas 2010; Lewis et al. 2011; Ma and Ma 2016; Orth et al. 2000; White et al. 2009). Effectors of this family have diverse and unique cellular functions in planta even within the same species (Lewis et al. 2011; Szczesny et al. 2010). AvrBsT is not a core effector but has a conserved sequence and is commonly found in $X$. perforans and some strains of $X$. vesicatoria and X. euvesicatoria (Minsavage et al. 1990; Potnis et al. 2011; Schwartz et al. 2015; Stall et al. 2009; Timilsina et al. 2016). X. euvesicatoria, X. vesicatoria, or $X$. perforans carrying avrBsT can cause an HR in nonhosts such as Arabidopsis thaliana Pi-0 and Nicotiana benthamiana (Cunnac et al. 2007; Escolar et al. 2001; Kim et al. 2010; Orth et al. 2000; Schwartz 
et al. 2015). Deletion mutants of avrBsT in X. vesicatoria (strain BV5-4a) produced mild symptoms in pepper when infiltrated at $5 \times 10^{4} \mathrm{CFU} / \mathrm{ml}$ (Kim et al. 2010). X. perforans race T4, group 2 strains carrying nonfunctional $a v r B s T$ were virulent on pepper when inoculated with a nonfunctional avrBsT (Schwartz et al. 2015). However, avrBsT deletion mutants in $X$. perforans group 1 strains did not cause disease in pepper, indicating additional factors restricting hostrange expansion in these strains (Schwartz et al. 2015). Furthermore, site-directed mutational analysis of the catalytic triad (His, Glu, and Cys) in the avrBsT gene abolished cell death but not effector delivery into plant cells (Cunnac et al. 2007; Orth et al. 2000). The catalytic triad in AvrBsT is essential for HR and cellular function and a single amino acid mutation in the catalytic triad abolishes HR ability in $N$. benthamiana and A. thaliana Pi-O (Orth et al. 2000). Kim et al. (2010) demonstrated the requirement of a full-length copy of avrBsT for inducing HR in pepper.

AvrBsT interacts with a number of effectors and host proteins in planta (Han and Hwang 2017; Szczesny et al. 2010). Unlike other XopJ-like effectors, AvrBsT is capable of interfering with AvrBs1induced $\mathrm{HR}$, indicating a direct interference with effector-triggered immunity (ETI) through a possible interaction with cell signaling factors such as SNF1-related kinase 1 (Szczesny et al. 2010). AvrBsT has acetyltransferase activity and acetylates the acetylated interacting protein 1 protein which is involved in ETI and pathogenassociated molecular pattern-triggered immunity responses (Cheong et al. 2014). AvrBsT downregulates defense-related genes (Senu4, Cevi16, and Tgas118) in tomato (Kim et al. 2010), whereas the HR in pepper is accompanied with induction of defense-related genes (CaBPR1, CaPO2, CaSAR82A, and CaDEF1), electrolyte leakage, and hydrogen peroxide accumulation (Kim et al. 2010). AvrXccb, an AvrBsT-homolog, suppressed immunity and interacted with cellular proteins in Brassica plants (Liu et al. 2017).

Pathogen fitness is ideally measured as the ability of a pathogen to survive and reproduce on a particular host (Laine and Barrès 2013; Leach et al. 2001). According to Vanderplank (1968), pathogens carrying unnecessary virulence genes have a fitness cost or penalty. Furthermore, mutations in avirulence genes occur to increase fitness in the presence of a resistance $(R)$ gene but can incur a fitness cost if an $R$ gene is not present (Vanderplank 1968). Fitness in plant-pathogenic bacteria can be measured by examining several

Table 1. List of bacterial strains and plasmids

\begin{tabular}{|c|c|c|}
\hline Strain or plasmid & $\begin{array}{c}\text { Relevant } \\
\text { characteristics }^{\mathrm{a}}\end{array}$ & Source or reference ${ }^{b}$ \\
\hline \multicolumn{3}{|l|}{ Xanthomonas perforans } \\
\hline GEV872 & Wild type; Rif ${ }^{R}$ & $\begin{array}{l}\text { Schwartz et al. } \\
2015 \text {; this study }\end{array}$ \\
\hline${\mathrm{GEV} 872 \mathrm{a} v r B s T \mathrm{del}_{572-615} \mathrm{fs}}$ & $\begin{array}{l}\text { avrBsT with an } \\
\text { internal deletion } \\
\text { and frameshift } \\
\text { mutant; Rif }{ }^{\mathrm{R}}\end{array}$ & This study \\
\hline GEV1001 & Wild type; Rif ${ }^{\mathrm{R}}$ & $\begin{array}{l}\text { Schwartz et al. } \\
2015 \text {; this study }\end{array}$ \\
\hline GEV1001 avrBsTdel $_{572-615} \mathrm{fs}$ & $\begin{array}{l}\text { avrBsT with an } \\
\text { internal deletion } \\
\text { and frameshift } \\
\text { mutant; Rif }{ }^{\mathrm{R}}\end{array}$ & This study \\
\hline \multicolumn{3}{|l|}{ Escherichia coli } \\
\hline DH5 $\alpha$ & $\mathrm{F}^{-}$rec $A$ & BRL \\
\hline$\lambda$ pir & Host for $\mathrm{pOK} 1 ; \mathrm{Sp}^{\mathrm{r}}$ & UB \\
\hline \multicolumn{3}{|l|}{ Plasmids } \\
\hline pGEM-T & $A m p^{R}$ & Promega Inc. \\
\hline pOK1 & $\begin{array}{l}\text { Suicide vector; } \\
\text { SacB }\end{array}$ & Huguet et al. 1998 \\
\hline pRK2013 & $\begin{array}{l}\text { Helper plasmid; } \\
\operatorname{Kan}^{\mathrm{R}}\end{array}$ & $\begin{array}{l}\text { Figurski and } \\
\text { Helinski } 1979\end{array}$ \\
\hline
\end{tabular}

${ }^{a} \operatorname{Rif}^{\mathrm{R}}, \mathrm{Sp}^{\mathrm{r}}, \mathrm{Amp}^{\mathrm{R}}$, and $\mathrm{Kan}^{\mathrm{R}}$ indicate resistant to rifampicin, spectinomycin, ampicillin, and kanamycin, respectively.

b $\mathrm{BRL}=$ Bethesda Research Laboratories and UB = University of Berkley. factors such as the rate of aggressiveness, infection efficiency, population growth, and survival under adverse environmental conditions (Leach et al. 2001). Several avirulence genes have been studied for their contribution to pathogen fitness in Xanthomonas spp. causing bacterial spot (Kearney and Staskawicz 1990; Leach et al. 2001). The highly conserved avirulence gene avrBs 2 contributes to pathogenicity and its deletion negatively affects fitness by reducing in planta growth of $X$. euvesicatoria (Kearney and Staskawicz 1990). In contrast, avrBs3 does not reduce bacterial populations and does not reduce aggressiveness but still contributes to pathogen fitness in the field (Wichmann and Bergelson 2004). Deletion of more than one effector gene in an $a v r B s 2$ mutant strain resulted in an additive fitness cost (Wichmann and Bergelson 2004).

Most studies focus on evaluating the effect of type III effectors on pathogen virulence under controlled environmental conditions. Therefore, a knowledge gap exists in corroborating greenhouse findings with actual field conditions. Furthermore, fitness studies examine pathogen fitness within the context of gene-by-gene interaction, yet the rate of discovering novel effector genes resulted in a shortage of discovering corresponding $R$ genes. As a result, using such $R$-gene-based approaches is inadequate or unavailable for evaluating specific and recently introduced effectors into a population. Nevertheless, the significance of possessing such effectors for pathogen virulence under field conditions remains crucial for disease management purposes. Therefore, in this study, we evaluated the effect of a type III effector, AvrBsT, under field conditions. We hypothesized that the ubiquitous presence of AvrBsT confers a selective advantage in the Florida $X$. perforans population and the loss of $a v r B s T$ should lead to a reduced pathogen fitness. For this purpose, we generated avrBsT deletion mutants in $X$. perforans strains representative of phylogenomic groups 1 and 2 and studied the effect of the $a v r B s T$ mutant strains on in planta growth, competition, movement, and epiphytic survival in comparison with the wild-type (WT) strain in tomato under field conditions.

\section{MATERIALS AND METHODS}

Bacterial strains, plasmids, and plants. $X$. perforans strains GEV872 and GEV1001 were collected during a survey in 2012 from commercial grower fields (Schwartz et al. 2015). Strains GEV872 and GEV1001 were selected as representative strains of $X$. perforans phylogenomic groups 1 and 2 (Schwartz et al. 2015). X. perforans GEV872 and GEV1001 were induced for rifampicin resistance by plating a $10^{8} \mathrm{CFU} / \mathrm{ml}$ bacterial culture of each strain on tryptone soy agar media amended with rifampicin at $200 \mathrm{mg} / \mathrm{ml}$. All GEV 872 and GEV1001 strains referred to hereafter are resistant to rifampicin. Plates were monitored over a period of 10 days and resistant colonies were selected and verified for rifampicin resistance. Antibiotics were added to media at the following concentrations: ampicillin at $100 \mu \mathrm{g} /$ $\mathrm{ml}$, rifampicin at $100 \mu \mathrm{g} / \mathrm{ml}$, kanamycin at $100 \mu \mathrm{g} / \mathrm{ml}$, spectinomycin at $100 \mu \mathrm{g} / \mathrm{ml}$, and cycloheximide at $100 \mathrm{mg} / \mathrm{ml}$. Bacterial strains, vectors, and plasmids used and constructed in this study are described in Table 1.X. perforans strains were cultured on nutrient agar (NA) or yeast glycerol agar at $28^{\circ} \mathrm{C}$ with or without amended antibiotics. Bacterial strains were cultured on NA at 28 and $37^{\circ} \mathrm{C}$ for $X$. perforans and Escherichia coli, respectively. Bacterial cultures used for inoculating plants were suspended in sterile tap water and the concentration was adjusted to $10^{8} \mathrm{CFU} / \mathrm{ml}$ (optical density at $600 \mathrm{~nm}=0.3$ ) using a spectrophotometer.

Tomato cultivar HM1823 (HM Clause, Davis, CA) was used in field trials conducted at the Gulf Coast Research and Education Center. Tomato cultivars HM1823 and FL47 (Seminis, St. Louis) were used for in planta growth curve and coinfiltration assays. Both tomato cultivars do not have any tolerance to bacterial spot. Pepper (Capsicum annuum L.) cultivar ECW, carrying the Bst resistance locus, was used for determining HR.

Mutant construction. X. perforans strain GEV872 was used as a template for amplifying the full-length $a v r B s T$ with primers $872 \mathrm{~F}$ 
(CAAACATGCCTTCGGTGA) and 872R (ATGTGCATAGTGC GGTGCAT). An amplicon of 1,858 bp, including 0.5-kb flanking regions, was cloned into pGEM-T Easy vector and sequenced using F20 and R24 primers for sequence confirmation. A 44-nucleotide internal gene deletion ( $a v r B s T_{572-615}$ ) was introduced into p-GEMT:avrBsT using internal primers with a HindIII unique restriction site (FBstDel: tatataagcttGGAGACATGGCGGTCATCCAAC and RBstDel: tatataagettAGCCGGCCATAACCTTAATTTCG) to produce p-GEM-T:avrBsTdel ${ }_{572-615}$ fs, resulting in a 12-amino-acid deletion and frameshift in the avrBsT open reading frame (ORF). The insert was sequenced, excised from the clone by digestion using restriction enzymes (ApaI/SpeI), and subcloned into the suicide vector pOK1 restricted with $A p a \mathrm{I} / X b a \mathrm{I}$ to produce pOK1: avrBsTdel $_{572-615}$ fs. Transformants were made in E. coli $\lambda$ pir. pOK1:avrBsTdel ${ }_{572-615} \mathrm{fs}$ was introduced into $X$. perforans by triparental mating using the helper plasmid pRK2013. Transformants carrying the mutated avrBsT were negatively selected using the $s a c B$ gene on NA plates amended with $5 \%$ sucrose and replicated on rifampicin-emended NA plates. Transformants with a complete double crossover that resulted in loss of pOK1 and, subsequently, $s a c B$ gene activity were selected from rifampicinamended plates. The avrBsT deletion was introduced into $X$. perforans GEV872 and GEV1001 by two-step homologous recombination and confirmation of mutants was made by polymerase chain reaction (PCR), sequencing, and restriction digestion of the avrBsT locus (Huguet et al. 1998). All strains were stored as a 20\% glycerol stock at $-80^{\circ} \mathrm{C}$.

In planta growth and competition assays. Strains were grown in nutrient broth (Difco Laboratories, Detroit) for $18 \mathrm{~h}$, harvested by centrifugation, and resuspended in sterile tap water or $0.01 \mathrm{M} \mathrm{MgSO}_{4}$. WT strains, mutant strains, and water were infiltrated into ECW pepper leaves at a concentration of $5 \times 10^{6}$ and $5 \times 10^{8} \mathrm{CFU} / \mathrm{ml}$. Four pepper leaves were infiltrated with each strain and water control at both concentrations and symptoms of $\mathrm{HR}$ were visually evaluated 1 to 3 days postinoculation (dpi). Pepper infiltration was conducted twice.

Bacterial suspensions of each strain were infiltrated separately in 4- or 6-week-old tomato FL47 or HM1823 seedlings at a concentration of $5 \times 10^{5} \mathrm{CFU} / \mathrm{ml}$. Three leaves in multiple plants were infiltrated with each X. perforans strain (GEV872:WT, GEV872: avrBsTdel ${ }_{572-615} \mathrm{fs}$, GEV1001:WT, and GEV1001:avrBsTdel ${ }_{572-615} \mathrm{fs}$ ). Following inoculation, plants were incubated at 24 to $28^{\circ} \mathrm{C}$. Three tomato leaflets were collected at a 24-h interval starting at 0 to $96 \mathrm{~h}$ after inoculation. Three leaf discs from inoculated leaves were collected for each infiltrated strain at each time point. Leaf discs $\left(1 \mathrm{~cm}^{2}\right)$ were individually macerated in $1 \mathrm{ml}$ of sterile tap water and a 10 -fold serial dilution was plated on NA media amended with rifampicin. Plates were incubated at $28^{\circ} \mathrm{C}$ and colonies were counted after 48 to $72 \mathrm{~h}$. CFU values were log-transformed and calculated as $\mathrm{CFU} / \mathrm{cm}^{2}$. In planta growth assays were repeated twice.

Furthermore, a comixture of $X$. perforans GEV872:WT + GEV872:avrBsTdel ${ }_{572-615}$ fs or X. perforans GEV1001:WT + GEV1001:avrBsTdel $572-615$ fs was infiltrated into 4-week-old tomato HM1823 seedlings at a $1: 1$ ratio and adjusted to $5 \times 10^{5} \mathrm{CFU} / \mathrm{ml}$. Leaf discs $\left(1 \mathrm{~cm}^{2}\right)$ were collected at 1,3 , and 5 or $1,3,5$, and $7 \mathrm{dpi}$ for the first and second assay, respectively. Two and three leaf discs were collected in the first and second assay, respectively. Leaf discs were processed as described above and diluted as necessary. Plates were incubated for $48 \mathrm{~h}$ at $28^{\circ} \mathrm{C}$. Eight single colonies from each leaf disc representative of the comixture were isolated, substreaked, and stored for further testing (see colony testing section).

Disease severity. $X$. perforans GEV872:WT, GEV872:avrBsTdel $_{572-615} \mathrm{fs}$, GEV1001:WT, and GEV1001:avrBsTdel ${ }_{572-615} \mathrm{fs}$ strains were individually spray inoculated until runoff on 4-weekold tomato seedlings at a concentration of $5 \times 10^{5}$ and $5 \times 10^{3} \mathrm{CFU} / \mathrm{ml}$. Three plants were inoculated per strain. Plants were bagged for $48 \mathrm{~h}$ after inoculation. Plants were monitored daily for disease development. At $12 \mathrm{dpi}$, three leaflets per strain were detached and evaluated for the area of disease using Assess 2.0 (American Phytopathological Society, St. Paul, MN).

Field trials. Three field trials were conducted during the 2015 (fall), 2016 (spring), and 2017 (spring) growing seasons at the Gulf Coast Research and Education Center in Balm, FL. Beds were fumigated, fertilized, sprayed with preplant herbicide, and covered with a white plastic mulch according to standard grower practices (Freeman et al. 2017). Gloves were changed between plots whenever touching plants was required to reduce cross contamination between plots. Plots were on 7.6-m-long beds with at least 15- to 21-m buffer zones between plots. Plants were obtained from a commercial grower. Tomato cultivars FL47 and HM1823 were used in the fall and springs seasons, respectively. Seedlings were transplanted at a 0.6-m distance within plots. Each plot contained 15 plants and treatments were set up in a randomized complete block design. The center plant of each plot was inoculated at a concentration of $5 \times 10^{6} \mathrm{CFU} / \mathrm{ml}$. Each plot was inoculated with either $X$. perforans GEV872 or GEV1001. During fall 2015, four leaves of the center plant in each plot were individually infiltrated with X. perforans GEV872:WT and GEV872:avrBsTdel ${ }_{572-615} \mathrm{fs}$, or with GEV1001:WT and GEV1001:avrBsTdel ${ }_{572-615} \mathrm{fs}$. In the spring 2016 and 2017 trials, center plants were dip inoculated into a suspension containing a mixture of GEV872:WT + GEV872: $\operatorname{avrBsTdel}_{572-615}$ fs or GEV1001:WT + GEV1001:avrBsTdel $572-$ ${ }_{615} \mathrm{fs}$, each adjusted to $5 \times 10^{6} \mathrm{CFU} / \mathrm{ml}$. The suspension also contained $0.05 \%$ (vol/vol) Silwett L77 (Helena, Collierville, TN). All trials included noninoculated control plots.

Disease severity was evaluated weekly and simultaneously with sample collection according to the Horsfall-Barratt scale. In fall 2015 , only symptomatic leaves were sampled every 7 days starting at 7 to 49 dpi. In spring 2016 and 2017, all leaves were sampled every 7 days starting at 7 to 70 dpi. Furthermore, noninoculated control plants were also sampled randomly throughout the trials. Four leaflets (approximately $2 \mathrm{~g}$ ) were sampled from the new growth to avoid redundant sampling. Leaves were washed in an extraction buffer (0.01 M MgSO $+0.2 \%$ Tween 20) (SigmaAldrich, St. Louis), and a 1-ml aliquot was sampled and diluted 10to 200 -fold. Only during the fall 2015 trial, the suspension was streaked using a sterile loop on unamended NA and rifampicinamended NA. During the spring 2016 and 2017 trials, a 50- $\mu \mathrm{l}$ aliquot was plated using the exponential slow deposition mode in an Autoplate 4000 Spiroplater (Spiral Biotech, Norwood, MA) on unamended NA and rifampicin-amended NA. Plates were incubated for 2 days at $28^{\circ} \mathrm{C}$. Bacterial concentrations were determined by counting colonies in the outer plate sectors in a grid, dividing the number of colonies by the volume deposited in the sector, and multiplying by the dilution factor for conversion into $\mathrm{CFU} / \mathrm{ml}$. The minimum detection threshold in our field trials was at $50 \mathrm{CFU} / \mathrm{ml}$ (approximately log 1.6). Plants with no CFU recovered were assigned the detection threshold for statistical analyses. Up to four single colonies, representative of each plant, were picked, substreaked, and stored for further colony testing.

Cleaved amplified polymorphic sequence PCR on rifampicin-resistant colonies. Colonies collected from rifampicin-amended plates of leaf washings were tested using cleaved amplified polymorphic sequence (CAPS)-PCR for the presence of the full-length or truncated avrBsT in WT or mutant strains, respectively. The maximum number of colonies tested for each plot was 60 , based on 4 colonies per 15 plants. DNA was extracted using the boiling method. Briefly, bacterial stocks of each single colony (total of approximately 6,000 ) were freshly streaked on NA amended with rifampicin. Using the tip of a loop, a small amount of pure bacterial growth was transferred into a $1.5-\mathrm{ml}$ microcentrifuge tube and resuspended in $400 \mathrm{ml}$ of sterile water. Tubes were boiled at $100^{\circ} \mathrm{C}$ for $15 \mathrm{~min}$, then immediately placed on ice for $5 \mathrm{~min}$, followed by centrifugation at $10,000 \mathrm{rpm}$ for $5 \mathrm{~min}$. A $2-\mu \mathrm{l}$ aliquot of the supernatant was used in PCR. Primers $872 \mathrm{~F}$ and $872 \mathrm{R}$ were used for amplifying the whole gene with flanking 
regions $(1,858 \mathrm{bp})$. PCR products were analyzed using agarose gel electrophoresis to confirm amplicon presence. Amplicons were then digested overnight using HindIII restriction enzyme and analyzed by electrophoresis on a $1 \%$ agarose gel. $X$. perforans colonies with a disrupted avrBsT ORF yielded two equal-sized bands (approximately 900 bp).

Statistical analyses. All statistical analysis were performed in SAS (v. 9.2; SAS Institute, Gary, NC). Generalized linear mixed models were employed using PROC GLIMMIX in SAS to determine the effect of treatments on response variables. Data were $\log$ transformed into a normal distribution prior to statistical analysis. Data were analyzed using a repeated measure model including a SPLICE function to compare treatments at individual intervals and to identify any treatment, time or time-treatment effect. Data were back-transformed and standard errors of the means were determined.

\section{RESULTS}

Loss of $\mathrm{HR}$ in pepper. To characterize the contribution of $a v r B s T$ to HR activity, pepper (ECW) carrying the BsT cognate resistance gene was infiltrated with four different $X$. perforans strains. $X$. perforans avrBsT mutants were generated in the background of two X. perforans strains, GEV872 and GEV1001. Leaves of pepper plants were infiltrated with WT strains of GEV872 and GEV1001. An HR was visible at 36 to $48 \mathrm{~h}$ after inoculation (Fig. 1A). Infiltrated areas were completely necrotic by $72 \mathrm{~h}$. On the same leaf, $X$. perforans strains GEV872:avrBsTdel ${ }_{572-615} \mathrm{fs}$ and GEV1001:avrBsTdel ${ }_{572-615}$ fs carrying an internal deletion of avrBsT did not elicit an HR response in ECW pepper plants carrying the cognate $B s t$ resistance gene. The absence of HR activity in pepper indicated loss of AvrBsT function in the mutant strains of GEV872 and GEV1001.

In planta growth in tomato leaf infiltration assays. The effect of $a v r B s T$ deletion was compared with the full-length $a v r B s T$ in different strains using bacterial growth curves. The growth curves for the WT and avrBsT mutant $X$. perforans strains were determined by infiltrating each strain separately in tomato leaves. In leafinfiltration assays, WT strains and mutant strains of GEV872 or GEV1001 did not show significant differences between populations (Fig. 1B). Populations initially started at approximately $3.4 \times 10^{4}$ and $2.7 \times 10^{4} \mathrm{CFU} / \mathrm{cm}^{2}$ and then increased by $96 \mathrm{~h}$ to $1.7 \times 10^{8}$ and $3.2 \times$ $10^{8} \mathrm{CFU} / \mathrm{cm}^{2}$ for GEV872:WT and GEV872:avrBsTdel ${ }_{572-615} \mathrm{fs}$, respectively. GEV1001:WT and GEV1001:avrBsTdel $572-615 \mathrm{fs}$ populations were initially at $2 \times 10^{4}$ and $5.5 \times 10^{4} \mathrm{CFU} / \mathrm{cm}^{2}$ and reached $4.2 \times 10^{7}$ and $4.98 \times 10^{7}$, respectively. Based on the growth curves herein, avrBsT does not affect bacterial populations sizes under these conditions.

In order to evaluate direct competition in planta, tomato leaves were coinfiltrated with rifampicin-resistant $X$. perforans strains
A

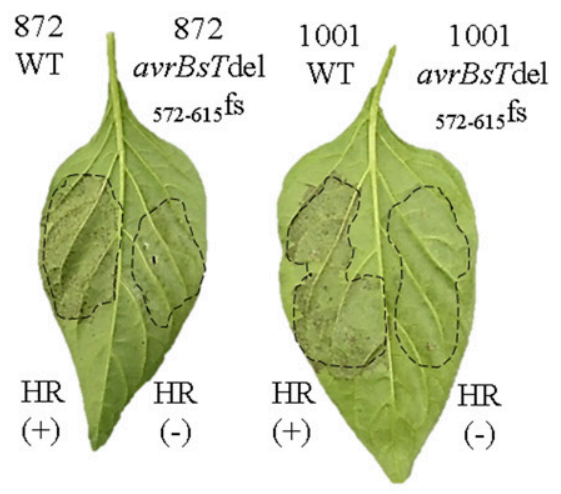

C

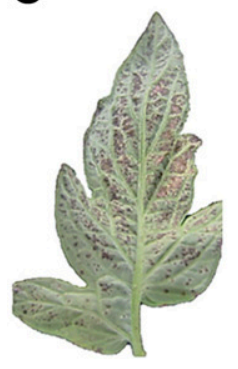

$872 \mathrm{WT}$

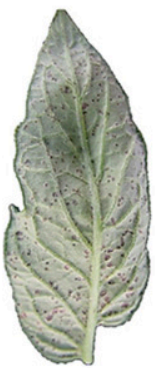

872 avrBsTdel ${ }_{572-615} \mathrm{fs}$

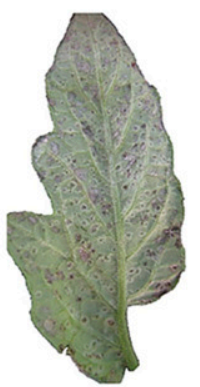

1001WT

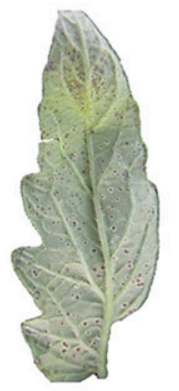

1001 avrBsTdel $572-615 \mathrm{fs}$
B

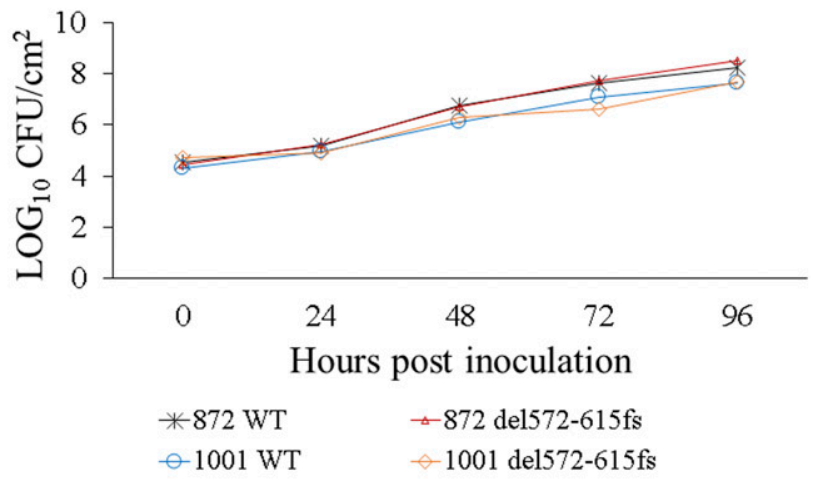

D

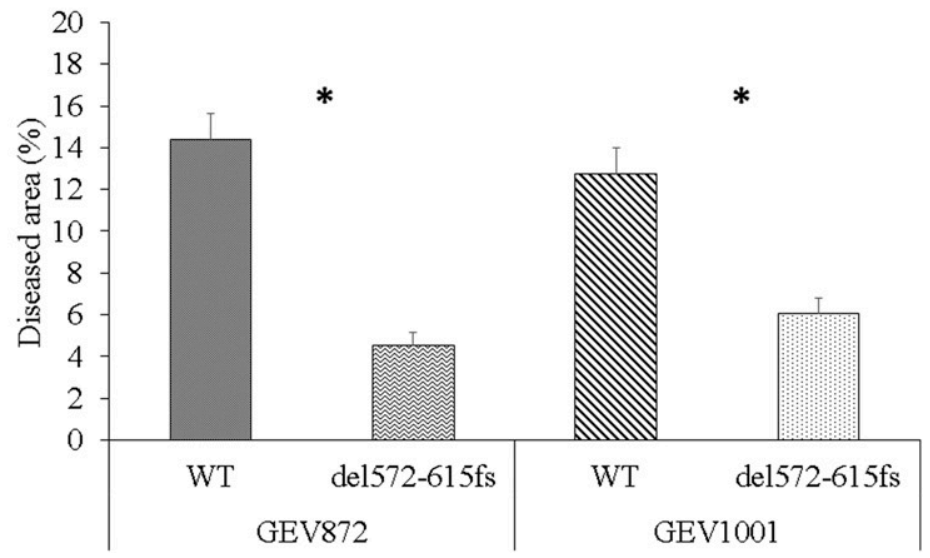

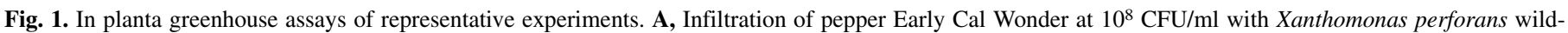

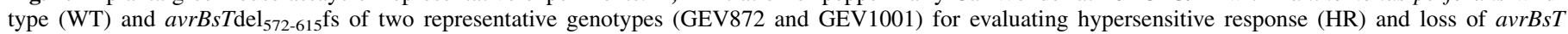

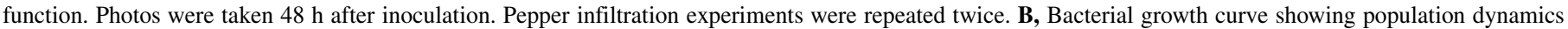

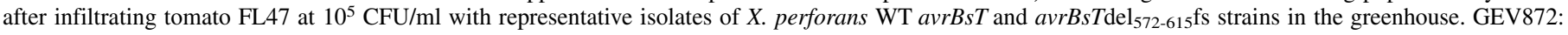

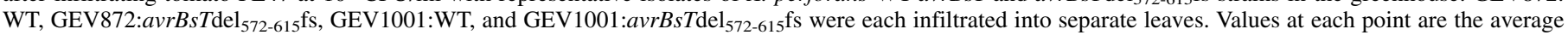

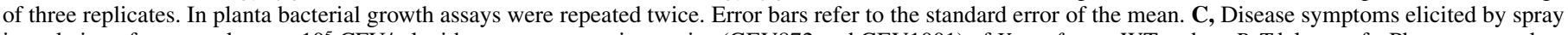

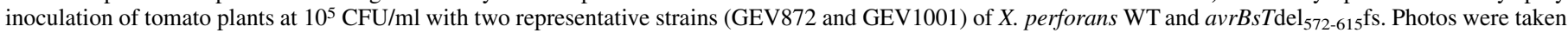

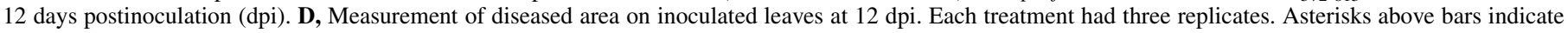
significant difference at $P<0.05$ between WT and mutants strains for GEV872 and GEV1001. 
GEV872WT + GEV872:avrBsTdel ${ }_{572-615}$ fs or GEV1001WT + GEV1001:avrBsTdel ${ }_{572-615}$ fs. Eight randomly selected colonies were tested from each coinfiltrated leaf disc by PCR and restriction digestion. Three leaves from three different plants were tested for the presence of WT or mutant strains of X. perforans GEV872 or GEV1001. Coinfiltration assays showed no statistical differences between WT strains and mutant strains of GEV872 or GEV1001 in three and two trials, respectively (data not shown). Only in one trial, the frequency of GEV1001:WT was two- to threefold higher $(P=$ 0.0227) than GEV1001:avrBsTdel ${ }_{572-615}$ fs. These results indicate the absence of direct competition between strains for survival.

Disease severity in greenhouse experiments. The disease severity of each strain was evaluated to assess whether disrupting avrBsT has an effect on symptom progression. Tomato seedlings were dip inoculated with $X$. perforans GEV872:WT, GEV872: $a_{\text {avrBsTdel }}{ }_{572-615} \mathrm{fs}$, GEV1001:WT, or GEV1001:avrBsTdel ${ }_{572-}$ ${ }_{615} \mathrm{fs}$ at $5 \times 10^{3}$ and $5 \times 10^{5} \mathrm{CFU} / \mathrm{ml}$. Water-soaked lesions were observed starting at 4 and $10 \mathrm{dpi}$ with plants inoculated with $10^{5}$ and $10^{3} \mathrm{CFU} / \mathrm{ml}$, respectively (Fig. 1C). Necrotic lesions became well defined by 7 and $15 \mathrm{dpi}$, respectively. At $12 \mathrm{dpi}$, we found the necrotic area of the leaf caused by GEV872:WT and GEV1001:WT greater than that of the strains lacking $\operatorname{avrBs} T$ (Fig. 1D). The average diseased leaf area was 14.4 and $4.5 \%$ for plants inoculated with GEV872:WT and GEV872: $a v r B s T \operatorname{del}_{572-615}$ fs, respectively, at $5 \times 10^{5} \mathrm{CFU} / \mathrm{ml}$. Similarly, the average diseased leaf area was 12.8 and $6.1 \%$ for strains GEV1001:WT and GEV1001:avrBsTdel ${ }_{572-615} \mathrm{fs}$, respectively. Similarly, plants infiltrated at a lower concentration of $10^{3} \mathrm{CFU} / \mathrm{ml}$ showed a similar trend; however, with an overall lower number of lesions and diseased area for both WTand $a v r B s T \mathrm{del}_{572-615} \mathrm{fs}$ strains (data not shown).

Disease severity and bacterial populations in field plants. Three field trials were conducted during fall 2015, spring 2016, and spring 2017 to evaluate the effect of $a v r B s T$ on several fitness factors. Center plants of each field plot were inoculated with a comixture of $X$. perforans GEV872:WT + GEV872: avrBsTdel ${ }_{572-615} \mathrm{fs}$ or GEV1001:WT + GEV1001:avrBsTdel ${ }_{572-615}$ fs. A noninoculated control plot was also included to monitor bacterial spread into neighboring plots. None of the noninoculated plots showed any disease throughout the season. Initially and later during the season, disease severity was highest on the center inoculated plants. Disease severity and bacterial population levels across the noninoculated plants within each plot were determined to monitor $X$. perforans infection and spread in the field. Representative data for disease severity and bacterial populations are presented for spring 2017. In spring 2017, disease severity of field plants initially increased to approximately $10 \%$ for strains GEV872 and GEV1001 during the first 2 weeks and then dropped to 2 to $4 \%$ over the next 6 weeks of the field trial. At $63 \mathrm{dpi}$, weather conditions became more conducive and disease severity rapidly increased to $55 \%$. Sharp increases in disease severity coincided with wet conditions. Disease severity and environmental conditions for all field trials are presented in Supplementary Figure S1.

Total bacterial populations of rifampicin-marked strains X. perforans. GEV872:WT + GEV872:avrBsTdel ${ }_{572-615} \mathrm{fs}$ or GEV1001:WT + GEV1001:avrBsTdel ${ }_{572-615} \mathrm{fs}$ were determined for each field plant to visualize bacterial spread across the field plots at a weekly interval. A subset of colonies representative of the bacterial population from each plant was stored and used for subsequent testing. Bacterial populations across trial plots initially exhibited a slightly bell-shaped distribution during the first few weeks, with the highest recovery of Xanthomonas levels occurring on plants within the center of the plot (Supplementary Fig. S2). Representative data from spring 2017 showed CFU levels of the center plants initially at $4.1 \times 10^{6}$ and $3.7 \times 10^{6} \mathrm{CFU} / \mathrm{g}$ of tissue but levels decreased within 14 days to $6.8 \times 10^{3}$ and $50 \mathrm{CFU} / \mathrm{g}$ of tissue for $X$. perforans GEV872 and GEV1001, respectively. Between 14 and $42 \mathrm{dpi}$, several plants had very low CFU levels and CFU values below the limit of detection except for the center inoculated plants.
At 21 and 28 dpi, GEV1001 was below the limit of detection and GEV872 was recovered at very low levels of $5 \times 10^{3} \mathrm{CFU} / \mathrm{g}$ of tissue. The recovery of GEV1001 and GEV872 increased by 49 dpi but was not significantly different from the levels during the first 2 weeks. At 56 dpi, CFU levels reached an average of $5.6 \times 10^{5}$ and $9.5 \times 10^{5} \mathrm{CFU} / \mathrm{g}$ of tissue for GEV1001 and GEV872, respectively. The recovery of both strains eventually plateaued after $56 \mathrm{dpi}$ and levels were not significantly different from each other. There was no significant interaction for bacterial populations between plant distance from center inoculated plant and time in spring 2016 or 2017. Bacterial populations from spring 2016 showed similar trends.

Effect of avrBsT on $X$. perforans movement in field plants. A subset of bacterial colonies from each plant within each plot was further tested for identification of WT or mutant strains for $X$. perforans GEV872 and GEV1001. The movement of $X$. perforans WT and $a v r B s T \mathrm{del}_{572-615}$ fs strains was evaluated as a function of incidence over distance and time across the plot to either side of the center inoculated plant. GEV872 and GEV1001 showed similar movement trends across each plot in each trial (Fig. 2A and B). Slight variations occurred in the overall movement of strains in each trial; however, the overall trend across all three field trials consistently showed that WT strains of either GEV872 or GEV1001 moved greater distances than the mutants for each $X$. perforans genotypes. During fall 2015, GEV872:WT was initially recovered at distances not significantly different than GEV872: avrBsTdel ${ }_{572-615} \mathrm{fs}$. At 21 to 28 dpi, GEV872:avrBsTdel ${ }_{572-615}$ fs surpassed GEV872: WT in distance but was not statistically different (data not shown). At 43 dpi, GEV872:WT was detected at a distance 1.2 times greater than GEV872:avrBsTdel $572-615$ fs, whereas GEV1001:WT was capable of moving across plots at greater distances and was significantly $(P=0.0004)$ different than GEV1001:avrBsTdel ${ }_{572-615}$ fs across all time points (data not shown). Furthermore, between 7 and 28 dpi, GEV1001:avrBsTdel ${ }_{572-615}$ fs did not readily spread across the plots. By 36 dpi, GEV1001:avrBsTdel ${ }_{572-615}$ fs movement lagged behind that of GEV1001:WT. GEV1001:WT spread a distance of 1.7 times greater than the mutant strain along the plots (data not shown).

In spring 2016, $X$. perforans GEV1001:WT moved greater distances across the plots compared with $X$. perforans GEV1001:

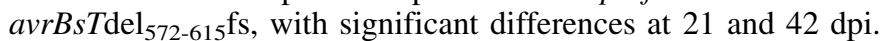
Furthermore, GEV872:WT was detected at a further distance than GEV872:avrBsTdel $572-615$ fs at each time period except $21 \mathrm{dpi}$ (Fig. 2A). Nevertheless, there were no statistical differences in movement between GEV872:WT and GEV872: avrBsTdel ${ }_{572-615} \mathrm{fs}$ strains over time (Fig. 2A). In spring 2017, both GEV872:WT and GEV1001:WT spread significantly $(P=0.0048$ and $P=0.0085$, respectively) further than both $a v r B s T$ del $_{572-615}$ fs strains (Fig. 2B). At 7 dpi, GEV872:WT and GEV1001:WT spread a distance of 4 and 1.1 times more than GEV872:avrBsTdel ${ }_{572-615} \mathrm{fs}$ and GEV1001:

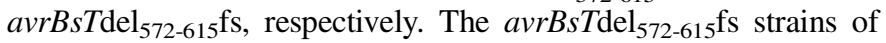
either genotype were not able to surpass the spread of the WT strains at any time period in the spring 2016 and 2017 trials and only at one time period during the fall 2015 trial. Statistical analysis did not show an interaction between time and treatments in all trials (Supplementary Tables S1 and S2).

Effect of avrBsT on strain recovery in field plants. For comparing the frequencies of WT and mutants strains in field plants, rifampicin-resistant colonies were recovered from the leaf surface of symptomatic or nonsymptomatic leaves. A subset of the rifampicin-resistant colonies recovered from leaf washings from each field plant was tested for the full-length or truncated $a v r B s T$ gene using CAPS-PCR. This subset included four colonies of rifampicin-marked $X$. perforans for each plant in the plot at a weekly interval. The number of colonies recovered from each plot varied due to fluctuations in disease severity and epiphytic populations. In total, more than 6,000 rifampicin-resistant colonies were tested from all three field trials. In all three trials, WT strains were recovered at a higher frequency compared with $\operatorname{avrBs} \mathrm{ddel}_{572-615} \mathrm{fs}$ strains. In fall 
2015, plots coinoculated with both WT and mutant strains showed that $X$. perforans GEV872:WT was recovered at a frequency significantly higher than $X$. perforans GEV872:avrBsTdel ${ }_{572-615}$ fs at $42 \mathrm{dpi}$ $(P=0.0052)$ and $49 \mathrm{dpi}(P=0.0219)$ but not at 14 to 35 dpi (data not shown). On the other hand, GEV1001:WT strains were recovered at a higher frequency than GEV1001:avrBsTdel ${ }_{572-615}$ fs $(P=0.0004)$ (data not shown).

In spring 2016 , there was a significant difference $(P=0.0001)$ between the frequency of WT and $a v r B s T$ del $_{572-615}$ fs strains of $X$. perforans GEV872 and GEV1001. At $21 \mathrm{dpi}$, frequency of GEV872:WT colonies were 1.1-fold higher compared with GEV872:avrBsTdel $572-615$ fs (Fig. 2C). However, after 28 dpi, the frequency of GEV872 WT colonies ranged between 2- and 4.4-fold higher than mutant strains. On the other hand, at $21 \mathrm{dpi}$, the frequency of GEV1001:WT colonies was 2.8-fold higher than GEV1001:avrBsTdel ${ }_{572-615}$ fs. GEV1001:WT decreased slightly to a 1.5 -fold difference by $35 \mathrm{dpi}$ and then increased to 3.1-fold difference after 56 dpi (Fig. 2C). A low number of colonies was tested at 42 dpi, coinciding with low total bacterial CFU (Fig. 2C).

In spring 2017, during the first 7 weeks, there was a low recovery rate of bacterial colonies (Fig. 2D), resulting in a low number of tested colonies for either WT or mutant strains. At 7 dpi, there was no significant difference between the frequency of WT or mutant strains. However, at $14 \mathrm{dpi}$, the frequency of $X$. perforans GEV1001:
WT strains was approximately twofold higher than the corresponding X. perforans GEV1001:avrBsTdel ${ }_{572-615}$ fs. At 21 and 28 dpi, GEV1001 was not recovered whereas GEV872 colony recovery was low (Fig. 2D). By 42 and 49 dpi, recovery of WT strains of each genotype was higher but not statistically different than the corresponding avrBsTdel ${ }_{572-615}$ fs strains. Recovery of $X$. perforans WT strains of GEV827 and GEV1001 significantly peaked at $56 \mathrm{dpi}$ to approximately five- and sevenfold, respectively, compared with avrBsTdel ${ }_{572-615}$ fs strains. A slight decrease followed at 63 and $70 \mathrm{dpi}$ in the frequency of WT colonies; however, the difference with

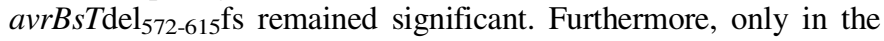
third trial (spring 2017), we recovered a very low number $(n=39)$ of rifampicin-resistant strains from the five noninoculated control plots at $70 \mathrm{dpi}$ (data not shown). The recovered colonies from noninoculated plots were dominantly carrying the full-length $a v r B s T$ gene $(n=37)$ whereas the remaining colonies carried a nonfunctional avrBsT. There was no interaction between time and treatments in all trials except for GEV1001 in spring 2017. The type III tests for fixed effects are included in Supplementary Tables S3 and S4).

\section{DISCUSSION}

Effector genes variably contribute to pathogen fitness and virulence levels. Effector genes are translocated into host cells by the T3SS
A

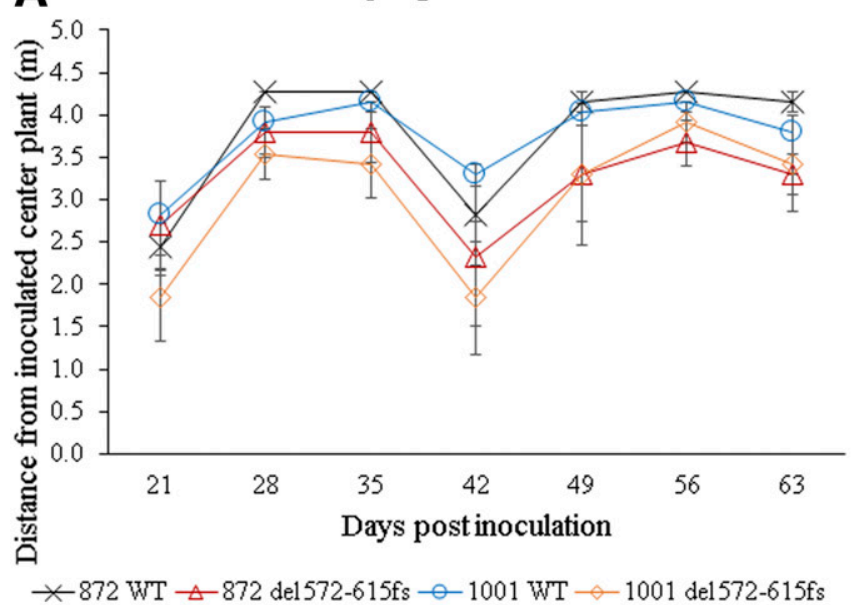

C

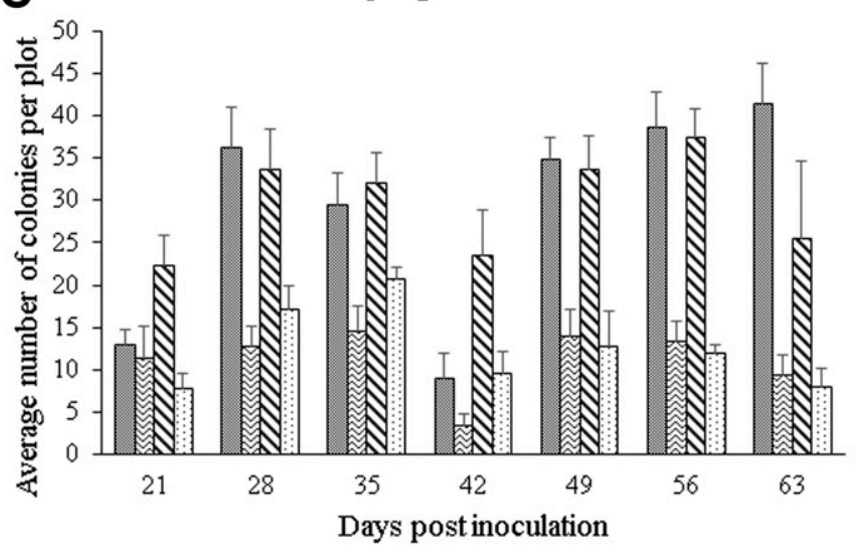

口872 WT @872 del572-615fs $\quad$ 1001 WT $\square 1001$ del572-615fs
B

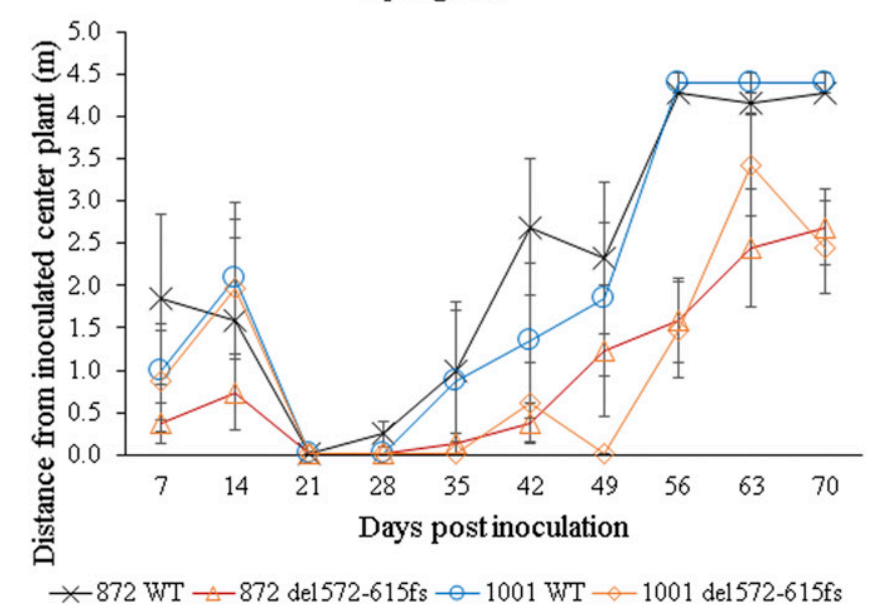

D

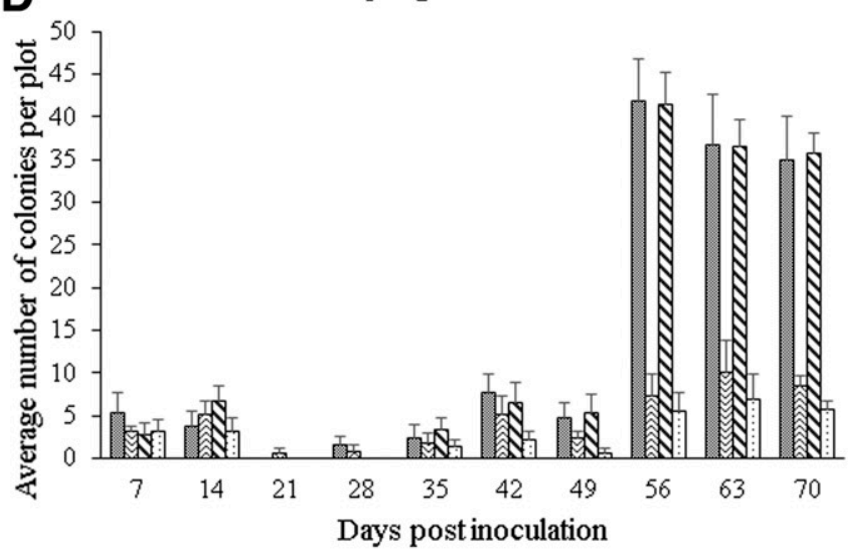

$\square 872 \mathrm{WT} \otimes 872$ del572-615fs $\quad$ 1001 WT $\square 1001$ del572-615fs

Fig. 2. Effect of $a v r B s T$ under field conditions. A, Recovery of Xanthomonas perforans GEV872 and GEV1001 wild-type (WT) and mutant (avrBsTdel ${ }_{572-615}$ fs) strains across field plots during 2016. B, Recovery of X. perforans GEV872 and GEV1001 WT and mutant (avrBsTdel ${ }_{572-615}$ fs) strains across field plots during 2017. C, Average number of colonies recovered for each WT and $a v r B s T d \mathrm{del}_{572-615} \mathrm{fs}$ strain during spring 2016. D, Average number of colonies recovered for each WT and $a v r B s T$ del $_{572-615}$ fs strain during spring 2017. Each data point is an average of five replicates. Each replicate contained 15 experimental units. Field trials were repeated three times and only results from two trials are presented in this figure. Error bars refer to the standard error of the mean. 
which is regulated by hrp genes. It is well documented that that deletion of hrp genes of the T3SS in most plant-pathogenic gramnegative bacteria leads to a dramatic decrease or abolishment of infection (Gürlebeck et al. 2006). Furthermore, several effectors were shown to contribute to pathogen fitness in planta or epiphytically under greenhouse conditions and very few under field conditions (Hirano et al. 1999; Leach et al. 2001; Vera Cruz et al. 2000; Wichmann and Bergelson 2004). In this study, we examined for the first time the effect of $a v r B s T$, an increasingly prevalent type III effector in the $X$. perforans population, on pathogen fitness at a large scale.

$X$. perforans mutant strains in this study were disrupted at the acetyltransferase catalytic domain of the gene, resulting in the abolishment of two essential amino acids responsible for cell death and auto- and transacetylation (Han and Hwang 2017). Therefore, based on our infiltration assays in pepper plants carrying the BsT resistance gene, our mutant strains did not elicit an HR, indicating gene inactivation and disruption of cellular catalytic activity. Comparison of population growth curves between strains carrying the full-length $a v r B s T$ gene and strains with a nonfunctional $a v r B s T$ gene did not affect bacterial growth in planta in the greenhouse. Therefore, $a v r B s T$ appears to be a nonessential virulence factor and does not have a fitness effect on in planta population under the conditions tested. Likewise, other studies showed that knocking out avrBs 1 and avrBs3 in X. euvesicatoria does not alter in planta population sizes but does have a fitness cost in the field (Wichmann and Bergelson 2004). However, this is in contrast to another study in which avrBsT deletion mutant strains of $X$. vesicatoria showed a lower population than the WT strain (Kim et al. 2010). The effect of AvrBsT on fitness might be species or strain specific but further studies are needed for confirmation. However, our results are in line with those described elsewhere for other XopJ-like effectors (Ciesiolka et al. 1999; Jiang et al. 2009; Noel et al. 2003). Deletion mutants of avrRxv (Ciesiolka et al. 1999) and $a v r X c c b$ (Jiang et al. 2009 ) in $X$. euvesicatoria and $X$. campestris pv. campestris and a frameshift deletion mutant of XopJ in X. euvesicatoria (Noel et al. 2003; Üstün et al. 2013) showed slight or no difference in bacterial populations compared with WT strains. Furthermore, a coinfiltration assay confirmed that there is no in planta interference between WT and $a v r B s T$ mutant strains. Evidence based on previous studies and within this study suggests that members of the XopJ family do not appear to have a significant effect on bacterial in planta growth.

Environmental conditions play a role in influencing pathogen population dynamics in the field. Xanthomonas spp. can spread between plant beds and throughout production fields by winddriven rain, irrigation water that is contaminated with the bacteria, and handling of plants (Pohronezny et al. 1990). Throughout the three trials, prolonged dry periods reduced rates of pathogen movement throughout the field; however, after periods of wetness, disease severity increased. Because plants were coinoculated simultaneously, disease severity was not distinguishable between WT and mutant strains. Disease severity levels were also similar across different time periods between the group 1 and group 2 strains, indicating a uniform level of disease development in the field. Although disease severity was low at specific time periods, bacterial population levels on plant leaves remained high. When epiphytic populations were examined in the field plants, we observed a strong trend in which mutant strains of either genotype had lower recovery levels than WT strains. The reduction in epiphytic populations of strains lacking certain effectors is not unusual. For example, $X$. campestris pv. malvacearum mutant strains with a nonfunctional avrb6 did not reduce pathogen population in planta; however, pathogen egress to the leaf surface was reduced (Yang et al. 1994). Similarly, avrBsT mutant strains appear to be significantly less prevalent as an epiphyte and, thus, disperse shorter distances than WT strains. As a result of higher epiphytic WT populations and conducive environmental conditions, WT strains were more readily disseminated across plants for both genotypes compared with strains lacking avrBsT.
Currently, there are no commercially deployed $R$ genes against $a v r B s T$ and, therefore, the presence of $a v r B s T$ should incur a fitness cost with no selective advantage in $X$. perforans. Nevertheless, under the conditions explored in these experiments, avrBsT provides a benefit to $X$. perforans with no associated cost. We showed that deploying avrBsT mutant strains in the field incurred a fitness cost apart from the presence of an $R$ gene. In this study, we used two genotypes representative of two phylogenomic groups and observed that $a v r B s T$ disruption in both genotypes is detrimental to fitness. Our data suggest that $a v r B s T$ might have a strain-dependent fitness cost; however, this needs to be confirmed in more strains of the same group. Strain-dependent virulence has been shown for avrPto, an effector in Pseudomonas syringae pv. tomato (Shan et al. 2000). Furthermore, it is worth noting that $X$. perforans group 2 strains might be more virulent compared with group 1, which might explain the higher prevalence of group 2 strains in Florida (P. Abrahamian, unpublished data). avrBsT is highly conserved and has no polymorphism based on recent comparative genomic studies in more than 100 strains (P. Abrahamian, unpublished data) (Schwartz et al. 2015; Timilsina et al. 2016). From a fitness cost perspective, the presence $a v r B s T$ should be dispensable for $X$. perforans but this is not the case for $a v r B s T$ based on our results in this work. The presence of this effector for almost 20 years in the $X$. perforans population makes this locus a good target in resistance breeding efforts. Sources of resistance to $a v r B s T$ were found in almost all pepper lines and in Solanum lycopersicoides and a line of $S$. lycopersicum (Wang 1992). Future work should focus on mapping the Bst locus in tomato and introgress the $R$ gene into commercially grown tomato cultivars. Pyramiding resistance genes against other conserved bacterial loci such as avrBs 2 and $a v r X v 4$ is essential to reduce resistance breakdown based on single-locus resistance.

\section{ACKNOWLEDGMENTS}

We thank R. Willis, S. Hughes, S. Kalb, K. Triner, C. Land, and A. Riniker for excellent technical assistance.

\section{LITERATURE CITED}

Alfano, J. R., and Collmer, A. 2004. TYPE III SECRETION SYSTEM EFFECTOR PROTEINS: Double agents in bacterial disease and plant defense. Annu. Rev. Phytopathol. 42:385-414.

Barak, J. D., Vancheva, T., Lefeuvre, P., Jones, J. B., Timilsina, S., Minsavage, G. V., Vallad, G. E., and Koebnik, R. 2016. Whole-genome sequences of Xanthomonas euvesicatoria strains clarify taxonomy and reveal a stepwise erosion of type 3 effectors. Front. Plant Sci. 7:1805.

Büttner, D., and Bonas, U. 2010. Regulation and secretion of Xanthomonas virulence factors. FEMS Microbiol. Rev. 34:107-133.

Cheong, M. S., Kirik, A., Kim, J.-G., Frame, K., Kirik, V., and Mudgett, M. B. 2014. AvrBsT acetylates Arabidopsis ACIP1, a protein that associates with microtubules and is required for immunity. PLoS Pathog. 10:e1003952.

Ciesiolka, L. D., Hwin, T., Gearlds, J. D., Minsavage, G. V., Saenz, R., Bravo, M., Handley, V., Conover, S. M., Zhang, H., Caporgno, J., Phengrasamy, N. B., Toms, A. O., Stall, R. E., and Whalen, M. C. 1999. Regulation of expression of avirulence gene $a v r R x v$ and identification of a family of host interaction factors by sequence analysis of avrBsT. Mol. Plant-Microbe Interact. 12:35-44.

Cunnac, S., Wilson, A., Nuwer, J., Kirik, A., Baranage, G., and Mudgett, M. B. 2007. A conserved carboxylesterase is a SUPPRESSOR OF AVRBSTELICITED RESISTANCE in Arabidopsis. Plant Cell 19:688-705.

Escolar, L., Van Den Ackerveken, G., Pieplow, S., Rossier, O., and Bonas, U. 2001. Type III secretion and in planta recognition of the Xanthomonas avirulence proteins AvrBs1 and AvrBsT. Mol. Plant Pathol. 2:287-296.

Figurski, D. H., and Helinski, D. R. 1979. Replication of an origin-containing derivative of plasmid RK2 dependent on a plasmid function provided in trans. Proc. Natl. Acad. Sci. USA 76:1648-1652.

Freeman, J. H., McAvoy, E. J., Boyd, N. S., Kinessary, R., Ozores-Hampton, M., Smith, H. A., Noling, J. W., and Vallad, G. E. 2017. Tomato production in Florida. Pages 329-372 in: Vegetable Production Handbook, Vol. HS739. G. E. Vallad, H. A. Smith, P. J. Dittmar, and J. H. Freeman, eds. University of Florida/IFAS, EDIS, Gainesville, FL. 
Galán, J. E., and Collmer, A. 1999. Type III secretion machines: Bacterial devices for protein delivery into host cells. Science 284:1322-1328.

Gürlebeck, D., Thieme, F., and Bonas, U. 2006. Type III effector proteins from the plant pathogen Xanthomonas and their role in the interaction with the host plant. J. Plant Physiol. 163:233-255.

Han, S. W., and Hwang, B. K. 2017. Molecular functions of Xanthomonas type III effector AvrBsT and its plant interactors in cell death and defense signaling. Planta 245:237-253.

Hirano, S. S., Charkowski, A. O., Collmer, A., Willis, D. K., and Upper, C. D. 1999. Role of the Hrp type III protein secretion system in growth of Pseudomonas syringae pv. syringae B728a on host plants in the field. Proc. Natl. Acad. Sci. USA 96:9851-9856.

Horvath, D. M., Stall, R. E., Jones, J. B., Pauly, M. H., Vallad, G. E., Dahlbeck, D., Staskawicz, B. J., and Scott, J. W. 2012. Transgenic resistance confers effective field level control of bacterial spot disease in tomato. PLoS One 7:e42036.

Huguet, E., Hahn, K., Wengelnik, K., and Bonas, U. 1998. hpaA mutants of Xanthomonas campestris pv. vesicatoria are affected in pathogenicity but retain the ability to induce host-specific hypersensitive reaction. Mol. Microbiol. 29:1379-1390.

Jacques, M.-A., Arlat, M., Boulanger, A., Boureau, T., Carrère, S., Cesbron, S., Chen, N. W. G., Cociancich, S., Darrasse, A., Denancé, N., Saux, M. F.-L., Gagnevin, L., Koebnik, R., Lauber, E., Noël, L. D., Pieretti, I., Portier, P., Pruvost, O., Rieux, A., Robène, I., Royer, M., Szurek, B., Verdier, V., and Vernière, C. 2016. Using ecology, physiology, and genomics to understand host specificity in Xanthomonas. Annu. Rev. Phytopathol. 54:163-187.

Jiang, W., Jiang, B.-L., Xu, R.-Q., Huang, J.-D., Wei, H.-Y., Jiang, G.-F., Cen, W.-J., Liu, J., Ge, Y.-Y., Li, G.-H., Su, L.-L., Hang, X.-H., Tang, D.-J., Lu, G.-T., Feng, J.-X., He, Y.-Q., and Tang, J.-L. 2009. Identification of six type III effector genes with the PIP box in Xanthomonas campestris pv. campestris and five of them contribute individually to full pathogenicity. Mol. Plant-Microbe Interact. 22:1401-1411.

Jones, J. B., Stall, R. E., and Bouzar, H. 1998. Diversity among xanthomonads pathogenic on pepper and tomato. Annu. Rev. Phytopathol. 36:41-58.

Kearney, B., and Staskawicz, B. J. 1990. Widespread distribution and fitness contribution of Xanthomonas campestris avirulence gene avrBs2. Nature 346:385-386.

Kim, N. H., Choi, H. W., and Hwang, B. K. 2010. Xanthomonas campestris pv. vesicatoria effector AvrBsT induces cell death in pepper, but suppresses defense responses in tomato. Mol. Plant-Microbe Interact. 23:1069-1082.

Laine, A. L., and Barrès, B. 2013. Epidemiological and evolutionary consequences of life-history trade-offs in pathogens. Plant Pathol. 62:96-105.

Leach, J. E., Cruz, C. M. V., Bai, J., and Leung, H. 2001. Pathogen fitness penalty as a predictor of durability of disease resistance genes. Annu. Rev. Phytopathol. 39:187-224.

Lewis, J. D., Lee, A. M. Y., Ma, W., Zhou, H., Guttman, D. S., and Desveaux, D. 2011. The YopJ superfamily in plant-associated bacteria. Mol. Plant Pathol. 12:928-937.

Liu, L., Wang, Y., Cui, F., Fang, A., Wang, S., Wang, J., Wei, C., Li, S., and Sun, W. 2017. The type III effector AvrXccB in Xanthomonas campestris pv. campestris targets putative methyltransferases and suppresses innate immunity in Arabidopsis. Mol. Plant Pathol. 18:768-782.

Ma, K.-W., and Ma, W. 2016. YopJ family effectors promote bacterial infection through a unique acetyltransferase activity. Microbiol. Mol. Biol. Rev. 80:1011-1027.

Minsavage, G., Dahlbeck, D., Whalen, M., Kearney, B., Bonas, U., Staskawicz, B., and Stall, R. 1990. Gene-for-gene relationships specifying disease resistance in Xanthomonas campestris pv. vesicatoria-pepper interactions. Mol. Plant-Microbe Interact. 3:41-47.

Noel, L., Thieme, F., Gäbler, J., Büttner, D., and Bonas, U. 2003. XopC and XopJ, two novel type III effector proteins from Xanthomonas campestris pv. vesicatoria. J. Bacteriol. 185:7092-7102.

Orth, K., Xu, Z., Mudgett, M. B., Bao, Z. Q., Palmer, L. E., Bliska, J. B., Mangel, W. F., Staskawicz, B., and Dixon, J. E. 2000. Disruption of signaling by Yersinia effector YopJ, a ubiquitin-like protein protease. Science 290:1594-1597.
Pohronezny, K., Moss, M. A., Dankers, W., and Schenk, J. 1990. Dispersal and management of Xanthomonas campestris pv. vesicatoria during thinning of direct-seeded tomato. Plant Dis. 74:800-805.

Pohronezny, K., Waddill, V., Schuster, D., and Sonoda, R. 1986. Integrated pest management for Florida tomatoes. Plant Dis. 70:96-102.

Potnis, N., Krasileva, K., Chow, V., Almeida, N. F., Patil, P. B., Ryan, R. P., Sharlach, M., Behlau, F., Dow, J. M., Momol, M., White, F. F., Preston, J. F., Vinatzer, B. A., Koebnik, R., Setubal, J. C., Norman, D. J., Staskawicz, B. J., and Jones, J. B. 2011. Comparative genomics reveals diversity among xanthomonads infecting tomato and pepper. BMC Genomics 12:146.

Potnis, N., Timilsina, S., Strayer, A., Shantharaj, D., Barak, J. D., Paret, M. L., Vallad, G. E., and Jones, J. B. 2015. Bacterial spot of tomato and pepper: Diverse Xanthomonas species with a wide variety of virulence factors posing a worldwide challenge. Mol. Plant Pathol. 16:907-920.

Schwartz, A. R., Potnis, N., Timilsina, S., Wilson, M., Patané, J., Martins, J., Jr., Minsavage, G. V., Dahlbeck, D., Akhunova, A., Almeida, N., Vallad, G. E., Barak, J. D., White, F. F., Miller, S. A., Ritchie, D., Goss, E., Bart, R. S., Setubal, J. C., Jones, J. B., and Staskawicz, B. J. 2015. Phylogenomics of Xanthomonas field strains infecting pepper and tomato reveals diversity in effector repertoires and identifies determinants of host specificity. Front. Microbiol. 6:535.

Shan, L., He, P., Zhou, J.-M., and Tang, X. 2000. A cluster of mutations disrupt the avirulence but not the virulence function of AvrPto. Mol. PlantMicrobe Interact. 13:592-598.

Stall, R. E., Jones, J. B., and Minsavage, G. V. 2009. Durability of resistance in tomato and pepper to Xanthomonads causing bacterial spot. Annu. Rev. Phytopathol. 47:265-284

Szczesny, R., Büttner, D., Escolar, L., Schulze, S., Seiferth, A., and Bonas, U. 2010. Suppression of the AvrBs1-specific hypersensitive response by the YopJ effector homolog AvrBsT from Xanthomonas depends on a SNF1related kinase. New Phytol. 187:1058-1074.

Timilsina, S., Abrahamian, P., Potnis, N., Minsavage, G. V., White, F. F., Staskawicz, B. J., Jones, J. B., Vallad, G. E., and Goss, E. M. 2016. Analysis of sequenced genomes of Xanthomonas perforans identifies candidate targets for resistance breeding in tomato. Phytopathology 106: 1097-1104.

Timilsina, S., Jibrin, M. O., Potnis, N., Minsavage, G. V., Kebede, M., Schwartz, A., Bart, R., Staskawicz, B., Boyer, C., Vallad, G. E., Pruvost, O., Jones, J. B., and Goss, E. M. 2015. Multilocus sequence analysis of Xanthomonads causing bacterial spot of tomato and pepper plants reveals strains generated by recombination among species and recent global spread of Xanthomonas gardneri. Appl. Environ. Microbiol. 81:1520-1529.

Üstün, S., Bartetzko, V., and Börnke, F. 2013. The Xanthomonas campestris type III effector XopJ targets the host cell proteasome to suppress salicylicacid mediated plant defence. PLoS Pathog 9:e1003427.

Vanderplank, J. E. 1968. Page 206 in: Disease Resistance in Plants. Academic, London, New York.

Vera Cruz, C. M., Bai, J., Oña, I., Leung, H., Nelson, R. J., Mew, T.-W., and Leach, J. E. 2000. Predicting durability of a disease resistance gene based on an assessment of the fitness loss and epidemiological consequences of avirulence gene mutation. Proc. Natl. Acad. Sci. USA 97: 13500-13505.

Wang, J. F. 1992. A new race of Xcv on tomato and screening tomato genotypes for resistance to this new race. Resistance to Xanthomonas campestris pv. vesicatoraia in tomato. Doctoral dissertation, University of Florida, Gainesville.

White, F. F., Potnis, N., Jones, J. B., and Koebnik, R. 2009. The type III effectors of Xanthomonas. Mol. Plant Pathol. 10:749-766.

Wichmann, G., and Bergelson, J. 2004. Effector genes of Xanthomonas axonopodis pv. vesicatoria promote transmission and enhance other fitness traits in the field. Genetics 166:693-706.

Yang, Y., De Feyter, R., and Gabriel, D. W. 1994. Host-specific symptoms and increased release of Xanthomonas citri and X. campestris pv. malvacearum from leaves are determined by the 102-bp tandem repeats of pthA and avrb6, respectively. Mol. Plant-Microbe Interact. 7:345-355. 